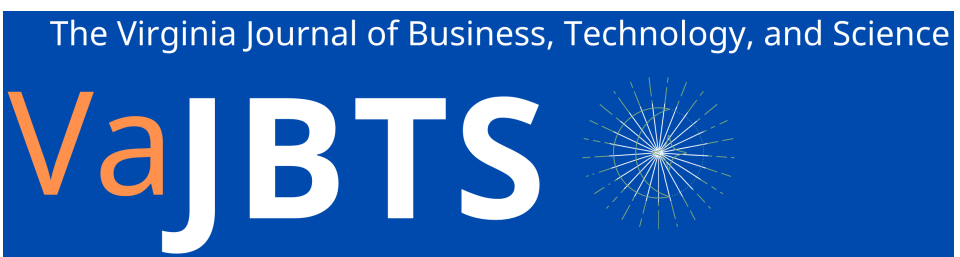

\title{
Research and development of scintillation detectors for the study of cosmic ray
}

\author{
Saikat Biswas ${ }^{1}$ \\ ${ }^{1}$ Department of Physics and Centre for Astroparticle Physics and Space Science (CAPSS), Bose Institute, EN-80, \\ Sector V, Kolkata-700091, India
}

July 6, 2021

\begin{abstract}
T Bose Institute Prof. Debendra Mohan Bose and his co-workers made globally recognised contributions A in the field of cosmic rays including the first recording of mu-meson tracks. Prof. D. M. Bose and Dr. Biva Choudhury did their cosmic ray experiments at the Darjeeling campus of Bose Institute (along with Sandakphu and Pharijong). Presently the Darjeeling campus hosts a National facility for Astroparticle Physics and Space Science. In Kolkata also there is a Centre for Astroparticle Physics and Space Science (CAPSS). In these two campuses, we are still working on the R\&D of plastic scintillation detectors for the study of the cosmic rays to preserve the legacy of Prof. D. M. Bose.

The only cosmic ray air shower array in the eastern part of India, consisting of seven plastic scintillator detectors is commissioned at an altitude of about 2200 meters above sea level (a.s.l.) in the Eastern Himalayas (Darjeeling) at the end of January 2018. The cosmic ray air shower array has a hexagon shape with six detectors kept at the vertices of the hexagon and one at the center of it. The distance between two consecutive detectors is 8 meters. Each detector element is made up of four plastic scintillators of dimension $50 \mathrm{~cm} \times 50 \mathrm{~cm} \times 1 \mathrm{~cm}$ thereby forming a total active area of $1 \mathrm{~m}^{2}$. These scintillators are fabricated indigenously in the Cosmic Ray Laboratory (CRL), TIFR, Ooty, India. All four scintillators of a detector are coupled with a single Photo Multiplier Tube (PMT) using wavelength shifting (WLS) fibers. A custombuilt module with seven inputs is used to generate a multi-fold trigger. Measurement of the number of cosmic ray air shower is going on since the end of January 2018. The secondary cosmic ray flux and its variation over time are also recorded at the laboratory in Darjeeling using a three-fold coincidence technique with plastic scintillators. All the details of the experimental setup, techniques of measurement are reported earlier. The updates in the results are presented in this article.

In this review article, the details of the $\mathrm{R} \& \mathrm{D}$ program of plastic scintillation detectors carried out during the last five years, for the study of cosmic ray is reported.
\end{abstract}

\section{Introduction}

Cosmic rays are high energy particles that mostly originate from outer space, with some very high energy particles even thought to have an extragalactic origin. Primary cosmic rays consist of $90 \%$ protons, $9 \%$ alpha particles, and other heavier nuclei. Secondary cosmic rays are created once primary cosmic rays react with the gas molecules in the atmosphere. These secondary particles consist mostly of pions and some kaons [I]]. Neutral pions $\left(\pi^{0}\right)$ decay into gamma rays that generate electromagnetic showers $\left(\mathrm{e}^{+}, e^{-}, \gamma\right)$ which are not very penetrating. Charged pions $\left(\pi^{+}, \pi^{-}\right)$decay into muons $\left(\mu^{+}, \mu^{-}\right)$and neutrinos $\left(\nu_{\mu}, \bar{\nu}_{\mu}\right)$. Neutrinos have a very small cross section for interaction and typically pass through the earth without any further interactions.

Cosmic rays lose energy as they travel through matter, mainly through ionisation. The Bethe-Bloch equation describes the energy loss of a particle due to ionisation.

$$
-\frac{d E}{d x}=\frac{4 \pi}{m_{e} c^{2}} \cdot \frac{n z^{2}}{\beta^{2}} \cdot\left(\frac{e^{2}}{4 \pi \epsilon_{O}}\right)^{2} \cdot\left\{\ln \left(\frac{2 m_{e} c^{2} \beta^{2}}{\left.I \cdot\left(1-\beta^{2}\right)\right)}-\beta^{2}\right\}\right.
$$




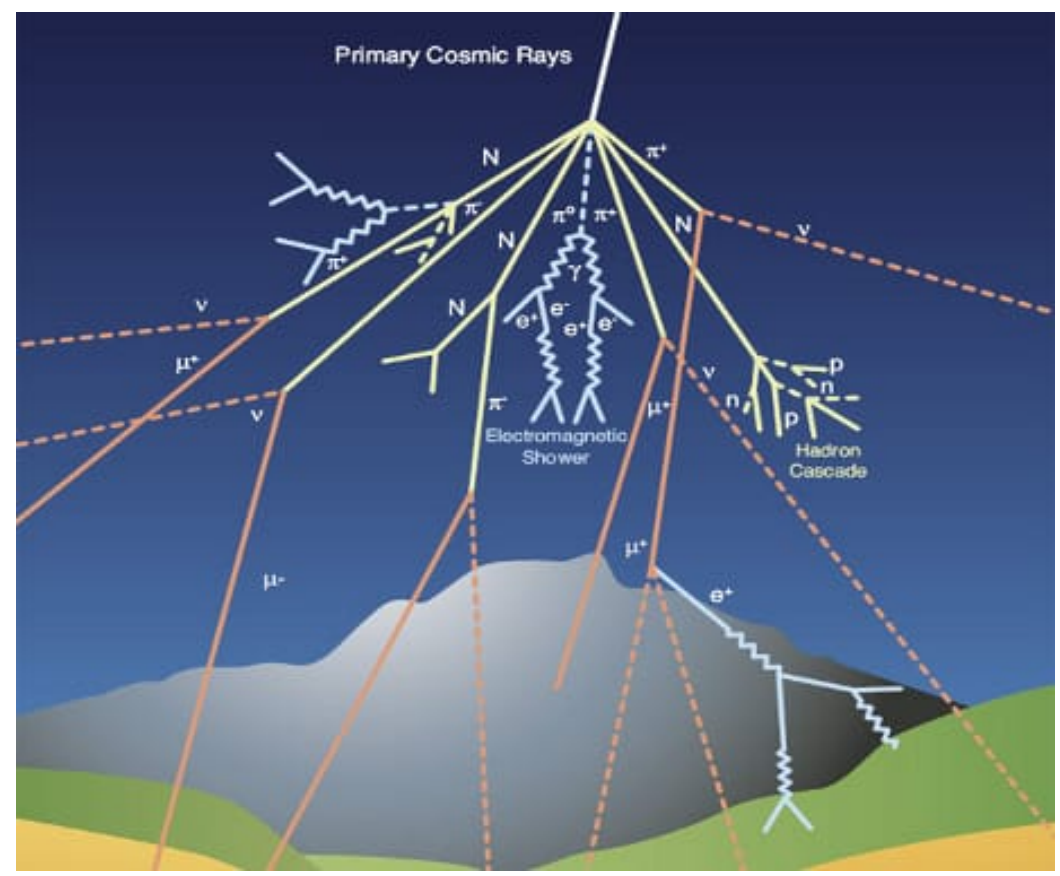

Figure 1: Cosmic Rays falling on earth

Radiation loss produced by the deceleration of a charged particle when deflected by another charged particle is a phenomenon called Bremsstrahlung. For a given momentum impulse, Bremsstrahlung is inversely proportional to the mass $^{2}$ of the radiating particle. The muon is essentially a heavy electron. It has the same spin and charge but the muon is $\sim 200$ times heavier than the electron. Muons are unstable; the positive muon $\left(\mu^{+}\right)$decays into a positron and two neutrinos, and the negative muon $\left(\mu^{-}\right)$decays into an electron and two neutrinos. Bremsstrahlung is negligible for muons due to its larger mass but crucial for electrons. This makes the muon a very penetrating particle unlike the electron. The muon has a lifetime of $2.2 \mu$ s yet it still makes it down to detectors at the surface of the earth penetrating the atmosphere $(480 \mathrm{~km}$ thick $)$. This is due to the fact that the muon travels close to the speed of light and so experiences relativistic time dilation, therefore we can detect them.

The cosmic ray energy spectrum has revealed new fine structures and consensus about the origin and composition of the primary particles has not yet been reached. Although an enormous amount of data has been obtained both through direct measurements by means of satellite or balloon borne experiments as well as indirect measurements such as Extended Air Shower (EAS) experiments to answer the questions about the

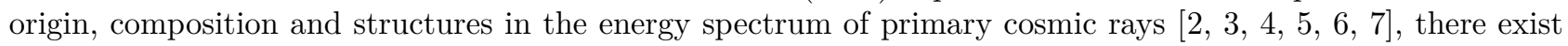
still a number of open questions on the above mentioned points. Various types of particle detection techniques including scintillation detectors of different types, muon tracking chambers, water and air Cherenkov detectors have been employed to detect the secondary particles produced in the air showers. One of the reasons for this is the lack of understanding of the hadronic interaction mechanisms during the course of primary cosmic rays through the atmosphere.

A new and simple technique is developed using plastic scintillator detectors for the study of angular variation of cosmic ray flux at different zenith angles near the sea level [ $[\mathbf{8}, \underline{\underline{9}}$.

Two scintillator detector modules using BC400 plastic are fabricated and tested with cosmic ray muons and different radioactive sources. As a proof of principle, a preliminary study is carried out using one paddle scintillator (named Sc-01) of dimension $20 \mathrm{~cm} \times 20 \mathrm{~cm}$ and one finger scintillator (named Sc-02) of dimension

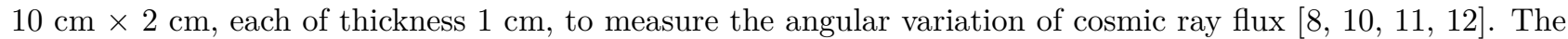
plastic scintillator material and the photomultiplier tube (PMT) are commercially procured. However, all other components such as the Perspex light guide, coupler for the light guide are fabricated in proper dimension at Bose Institute workshop. In this experiment, the conventional NIM electronics is used. The data analysis is carried out using the ROOT software package [1]3].

On the other hand, an array of plastic scintillation detectors is proposed for the detection of cosmic ray show- 
ers at an altitude of about 2200 meters a.s.l. in the Eastern Himalayas at the National facility for Astroparticle Physics and Space Science, Darjeeling campus of Bose Institute. Each element of this array is a $1 \mathrm{~m} \times 1 \mathrm{~m}$ plastic scintillation detector of thickness $1 \mathrm{~cm}$, coupled with WLS fibers and a PMT. Seven of these modules arranged in a hexagonal way keeping one at the centre of the hexagon is built and commissioned in the beginning of 2018, during the first phase of the project. This detector array is constructed for a better understanding of the dependence of cosmic ray fluxes on the geographical parameters such as latitudes, longitudes, altitude, etc. The main goal of this program is to study the properties of cosmic rays at high altitude. The method of fabrication of the detectors, experimental set-up, and the preliminary results are discussed in detail in Refs. [14] and [15]. We have carried out the measurements for a longer time duration to increase the statistics of the result in our earlier work. The updated result is presented in Ref. [16].

This particular review article is based on the R\&D activities carried out in the Centre for Astroparticle Physics and Space Science (CAPSS), Kolkata and National facility for Astroparticle Physics and Space Science, Darjeeling of Bose Institute on scintillation detectors during the last five years [9, 14, 15, 16].

\subsection{Plastic Scintillator}

Scintillators are materials that are able to convert energy deposited by high energy charged particles like muons, pions etc. into photons of near visible region. They are of gaseous, liquid or solid materials and either organic or inorganic. The requirements that a scintillator detector should have are:

1) High efficiency for conversion of exciting energy to fluorescent radiation.

2) Transparency to its fluorescent radiation.

3) Emission in a spectral range consistent with the spectral response of existing photomultipliers.

4) Short decay constant.

Plastic scintillator is one of the most widely used detector to detect particles produced in high-energy physics experiments because of its large area coverage. It is a device with scintillating material coupled with an amplifier to convert scintillation light into electrical signal and can be counted or analysed. It offers an extremely fast signal. The main advantage of these detectors is their flexibility, large area coverage, easy to fabricate and operate and as they can be made of any size or shape. The main purpose of our R\&D with this kind of scintillator is to study the physics of cosmic ray and also to use it as trigger for other experiments.

Plastic scintillators have a relatively short response time which is typically of the order of a few nanoseconds. Plastic scintillators are reliable, robust, and convenient [7, [8]. However, they possess quirks to which the experimentalists must be careful. Exposure to solvent vapours, high temperatures, mechanical flexing, irradiation, or rough handling will adverse the processes. A particularly fragile region is the surface which can develop micro-cracks which degrade its transmission of light by total internal reflection. A charged particle traversing these plastic material leaves behind some excited molecules. However, certain types of molecules will release a small fraction (about 3\%) of this energy as optical photons. This process, scintillation, is especially marked in those organic substances which contain aromatic rings, such as polystyrene (PS) and polyvinyl toluene (PVT). The scintillator material used in this experiment has PVT as its base and contains $65 \%$ anthracene. A scintillator detector has three main components:

1) Scintillator material

2) Light guide

3) Photomultiplier tube (shown in Figure 『).

\subsection{Photomultiplier tube (PMT)}

Photomultipliers (PMs) are devices that convert light into a measurable electric current. They are extremely sensitive and mostly used in nuclear and high-energy physics experiments. Figure $\square$ shows a schematic diagram of a typical photomultiplier. It consists of a cathode made of photosensitive material followed by an electron collection system, an electron multiplier section (or dynode string as it is usually called) and finally an anode from which the final signal can be taken. All parts of PMT are usually housed in an evacuated glass tube. During operation a high voltage (HV) is applied to the cathode, dynodes and anode such that a potential "ladder" is set up along the length of the cathode - dynode - anode structure. When an incident photon (from a scintillator for example) impinges upon the photocathode, an electron is emitted via the photoelectric effect. Because of the applied voltage, the electron is then directed and accelerated toward the first dynode, whereupon striking, it transfers some amount of its energy to the electrons in the dynode. This causes secondary electrons to be emitted, which in turn, are accelerated towards the next dynode where more electrons are released and 


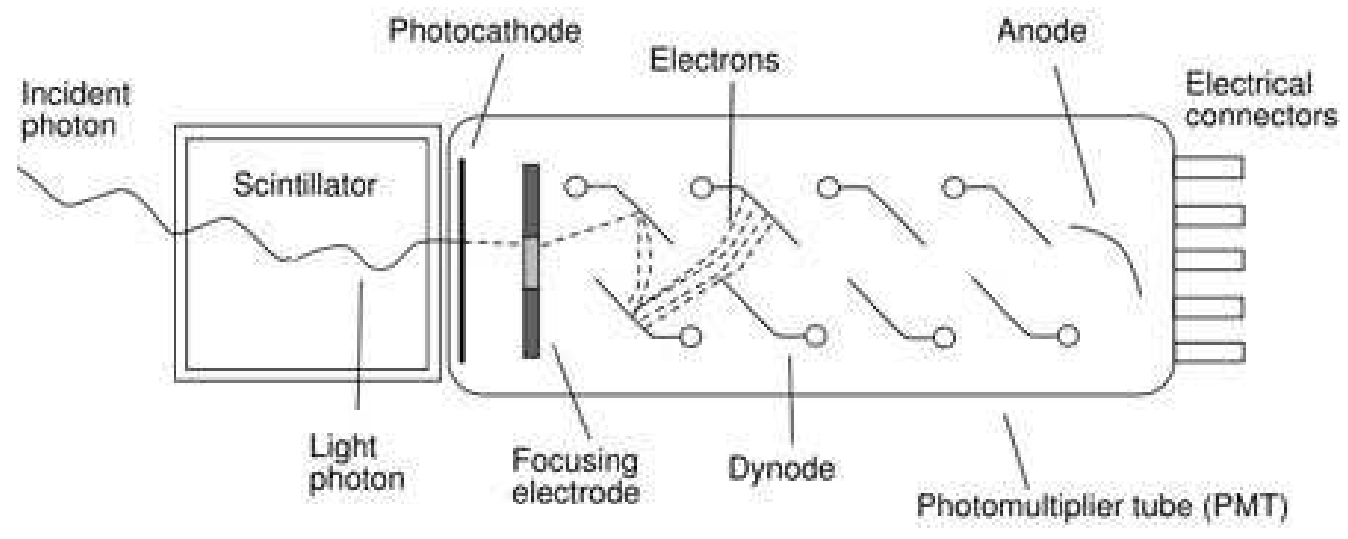

Figure 2: Main Parts of the plastic scintillator

further accelerated. An electron cascade down the dynode string is thus created. At the anode, this cascade is collected to give a current which can be amplified and analysed.

\section{Measurement of angular variation of cosmic ray intensity}

A new and simple technique is developed using plastic scintillator detectors for the study of angular variation of cosmic ray intensity near the sea level. The motivation of this work is to measure the cosmic ray flux at different zenith angles, nearly at the sea level (in Kolkata, India during the beginning of 2017), using plastic scintillation detectors. The details of the fabrication of the scintillator modules are described in Sec [2.], experimental set-up and results are shown in Sec [2.2 and Sec 2.3 respectively.

\subsection{Fabrication of the scintillator modules}

One BC400 type $40 \mathrm{~cm} \times 40 \mathrm{~cm}$ scintillator plate of thickness $1 \mathrm{~cm}$ is procured. At Bose Institute workshop this plate is cut into several small pieces to build the detectors.

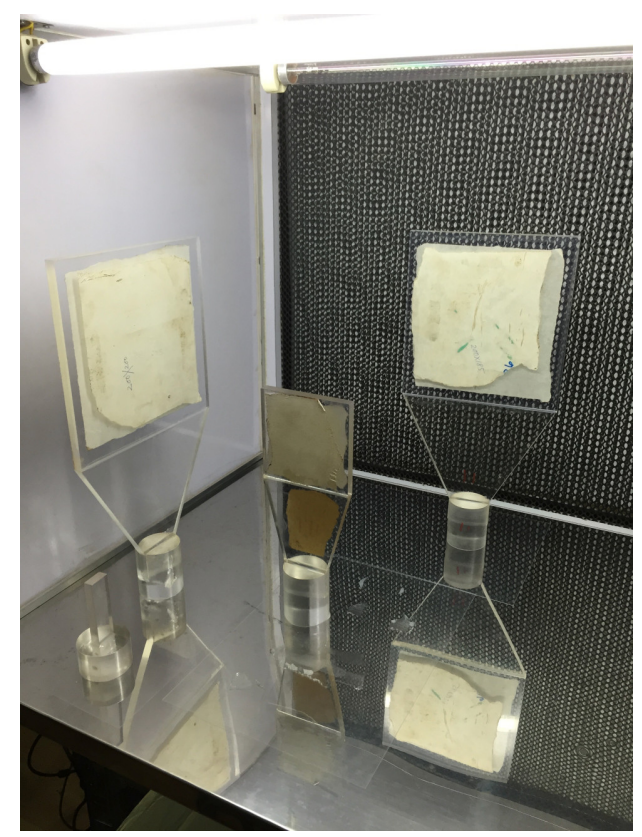

Figure 3: Gluing of scintillators with the light guide 
In this particular study one plastic scintillator paddle detector having dimension $20 \mathrm{~cm} \times 20 \mathrm{~cm}$ and one finger scintillator detector of size $10 \mathrm{~cm} \times 2 \mathrm{~cm}$ are used. For the scintillator paddle, the Perspex light guide is trapezoidal in shape whose longer side (connected to the scintillator) is $20 \mathrm{~cm}$, shorter side (connected to one end of the cylindrical light guide) is $5.1 \mathrm{~cm}$ and length about $15 \mathrm{~cm}$. The other side of the cylindrical light guide also made of Perspex is connected to the PMT of diameter $5.1 \mathrm{~cm}$.
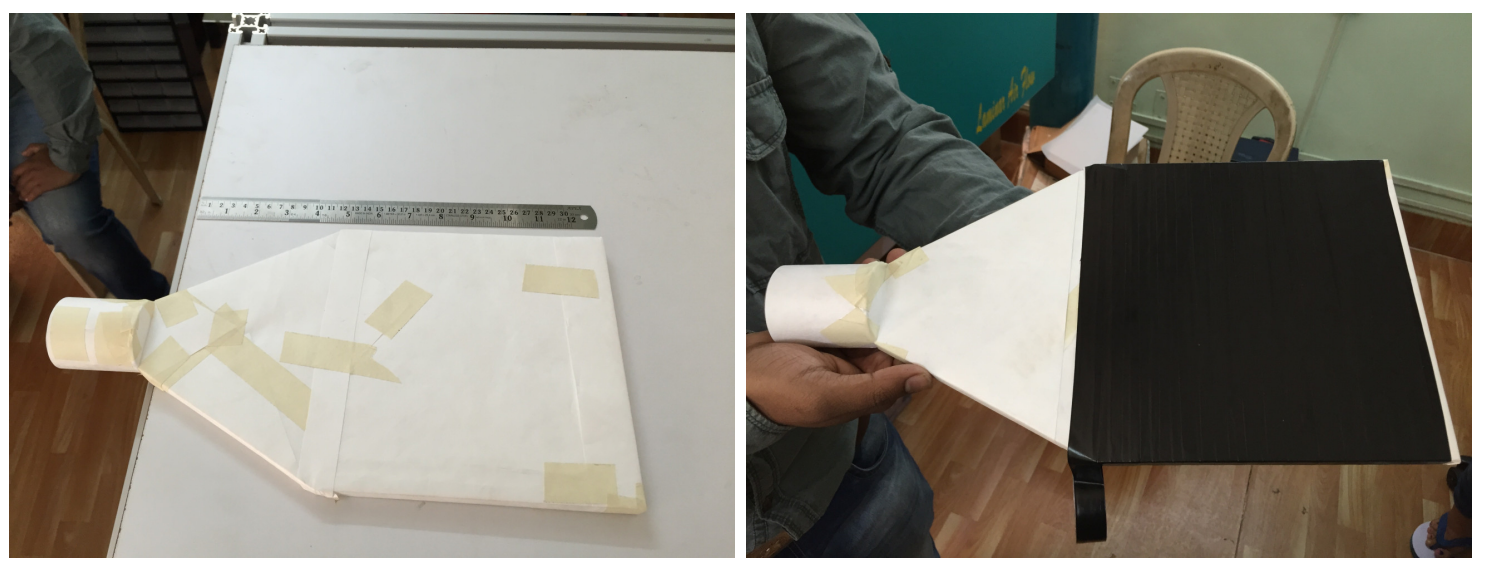

Figure 4: Wrapping with Tyvek paper (left) and with black tape (right)

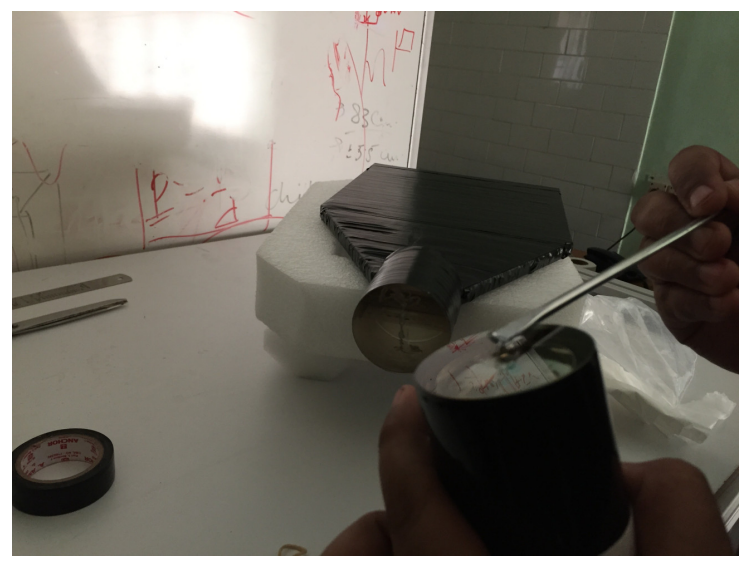

Figure 5: Application of optical grease to PMT

The plastic scintillator BC-400 is glued with the light guide using BC-600 optical cement and hardener in the ratio $5: 1$, which is a clear epoxy resin that sets at room temperature and has a refractive index close to that of our premium plastic scintillators. After gluing the different components, the scintillator modules are left for 24 hours at room temperature for curing as shown in Figure [3. After curing the entire thing is wrapped tightly with Tyvek paper using paper tapes and then black tapes are wrapped around the scintillator as shown in Figure 由. The PMT is joined to the cylindrical light guide using optical grease as shown in Figure 1 . The entire assembly of the scintillator and PMT is wrapped with black tapes twice to ensure minimum light leakage. Then the base voltage divider circuit is connected to the PMT. In the base, there is one SHV cable with a connector for the application of high voltage and one BNC cable with a connector for the signal. The base voltage divider circuit and the pins of the PMT are shown in Figure $\mathbf{6}$. The complete scintillator paddle detector is shown in Figure $\mathbf{7}$. In total two paddle detectors of dimension $20 \mathrm{~cm} \times 20 \mathrm{~cm}$, one paddle of dimension 10 $\mathrm{cm} \times 10 \mathrm{~cm}$ and one finger of size $10 \mathrm{~cm} \times 2 \mathrm{~cm}$ are built. 


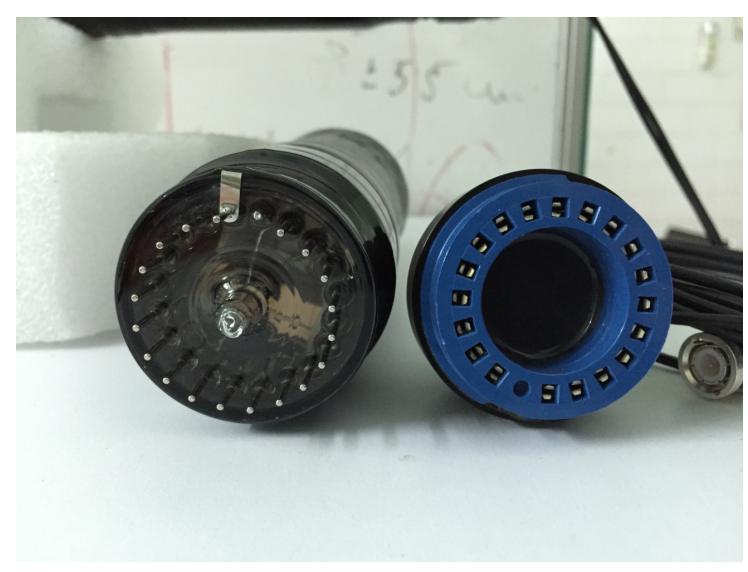

Figure 6: PMT and base of voltage divider

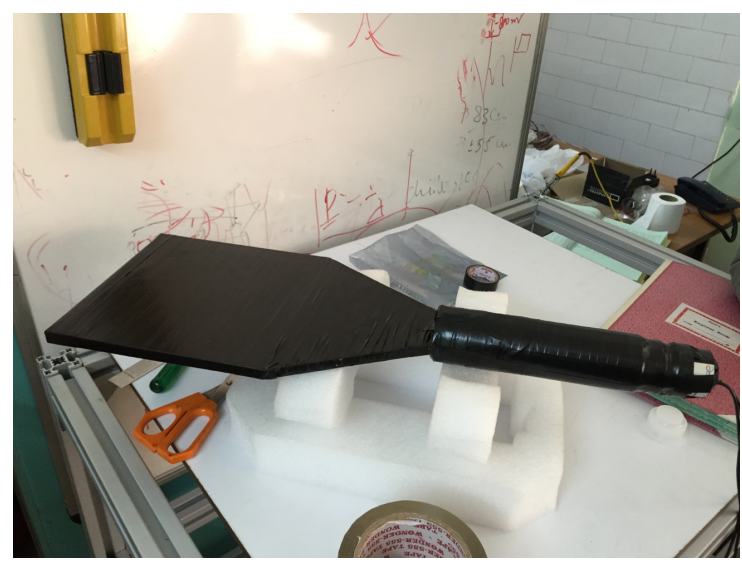

Figure 7: Completed scintillator paddle detector

\section{$2.2 \quad$ Experimental Set-up}

In this experiment, the detectors are placed in a specialised and adjustable aluminium rack where both horizontal (X-axis) and vertical (Y-axis) movements are easily possible. To measure the cosmic ray flux at various zenith angles, the paddle scintillator is kept fixed at a particular position and the position of the finger detector is changed. The schematic of the experimental set-up is shown in Figure $\mathbf{8}$.

A bias voltage of $1650 \mathrm{~V}$ is applied to both the scintillators. The signals from the scintillation detectors are fed to a leading edge discriminator (LED). Thresholds to the LED are set at $-50 \mathrm{mV}$. The discriminated NIM signals are put to a logic unit and the coincidence count is measured using a NIM counter.

Initially, the scintillators are calibrated to get their operating voltages. The PMTs are calibrated by measuring the count rates of the scintillator paddle and the finger varying the applied voltages to the PMT from $1000 \mathrm{~V}$ to $1850 \mathrm{~V}$. The thresholds to the LED are set at $-50 \mathrm{mV}$. For each voltage settings count rates are measured using three different radioactive sources e.g. $\mathrm{Cs}^{137}, \mathrm{Na}^{22}, \mathrm{Co}^{60}$. The background count is taken for each voltage setting and it is subtracted from the count taken with the source. It is observed that for each radioactive source the count rate increases with voltage and reaches a plateau for voltage $\sim 1600 \mathrm{~V}$ and onwards. So the bias voltage to both the detectors are set at $1650 \mathrm{~V}$ for all the measurements.

Yield uniformity is also checked using $\mathrm{Co}^{60}$ source. The active area of the scintillator Sc-01 is divided in 64 imaginary grids each of area $2.5 \mathrm{~cm} \times 2.5 \mathrm{~cm}$. The $\mathrm{Co}^{60}$ source is placed at the centre of each grid and counts are taken for a time duration of 60 seconds. Though the yield is uniform in the central region, there is a variation of $12 \%$ in the entire scintillator.

For the measurement of angular variation of cosmic ray intensity, the $\mathrm{X}$-axis is chosen to be parallel to the east-west direction. The number of coincidence signals is counted for different angles of incidence of the cosmic 


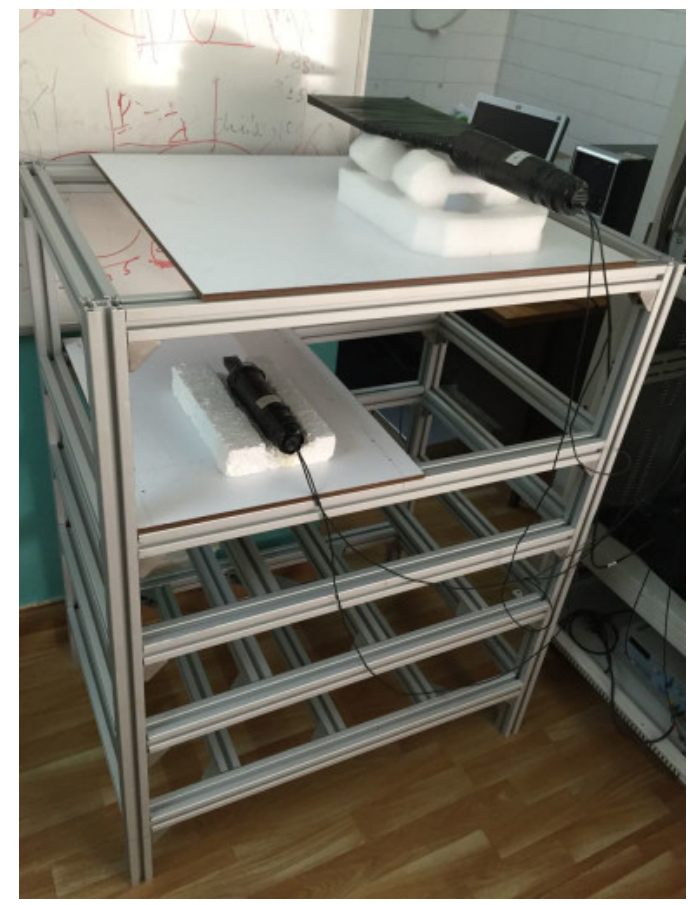

Figure 8: Experimental set-up to study angular variation of cosmic ray flux

ray muons for a time duration of 60 minutes each. The Sc-02 module is kept below the Sc-01 along the vertical direction. Sc-02 is moved in the horizontal plane and the coincidence counts are taken at different positions of Sc-02 (along X-axis). Thus the incident cosmic ray flux within the solid angle subtended by the modules Sc-01 at Sc-02 for a particular angle of incidence is measured. This measurement is performed for different zenith angles. An expression for the solid angle for this particular experimental setup is derived and the approximate value in steradian is calculated for a given zenith angle using a 2D integration program in ROOT. The coincidence count rate for different zenith angles of cosmic rays is normalised by the solid angle. The same process is repeated with different perpendicular separation between Sc-01 and Sc-02. The singles count for both the detectors are also measured in each set of readings to check the consistency of data. No remarkable variations in singles count are observed. The cosmic ray shower rate, measured keeping two scintillators at a separation of $3 \mathrm{~m}$ is found to be $\sim 10^{-5}$ per second per unit area and is subtracted from all data points.

\section{$2.3 \quad$ Result}

In this study, the measured quantity is the coincidence count rate from the detectors. To measure the angular variation of the cosmic ray flux, the detectors are moved as described in Sec [2.2. Firstly the detector Sc-02 is kept on the lower rack just below the Sc-01 scintillator as shown in Figure $\mathbb{Q}$ This position of Sc-02 is marked as the origin of the X-axis. The coincidence count rates of Sc-01 and Sc-02 are measured for this arrangement. This reading corresponds to the cosmic rays that are vertically incident on our setup. Sc-02 is moved $5 \mathrm{~cm}$ along positive $\mathrm{X}$-axis and measurements are repeated. This reading corresponds to the cosmic rays incident at a zenith angle given by $\theta=\tan ^{-1}(5 / y)$ in degrees, where y is the perpendicular separation of the modules Sc-01 and Sc-02. Measurements are repeated by moving Sc-02 in a step of $5 \mathrm{~cm}$ along X-axis. Thus coincidence count rates for different zenith angle is measured. The same process is carried out by moving Sc-02 along negative $\mathrm{X}$-axis. The average of the coincidence counts of both negative and positive X-axis is taken. A second method to measure the angular variation of cosmic rays is also used. In this method, the position of Sc-01 is kept fixed, and Sc-02 is moved vertically along Y-axis keeping the separation between Sc-01 and Sc-02 along X-axis same. First Sc-01 and Sc-02 are kept on the same plane (XZ plane) and coincidence counts of Sc-01 and Sc-02 is measured. This count corresponds to the count for cosmic rays traveling horizontally. Then, the scintillator Sc-02 is moved downwards along Y-axis and coincidence counts are measured at various such y positions. The cosmic ray showers incident on our set up and also the chance coincidence counts are found to be negligible. The solid angle $\Omega(\theta)$ subtended by Sc-01 at Sc-02 is also calculated for each of such cosmic ray incidence angles 


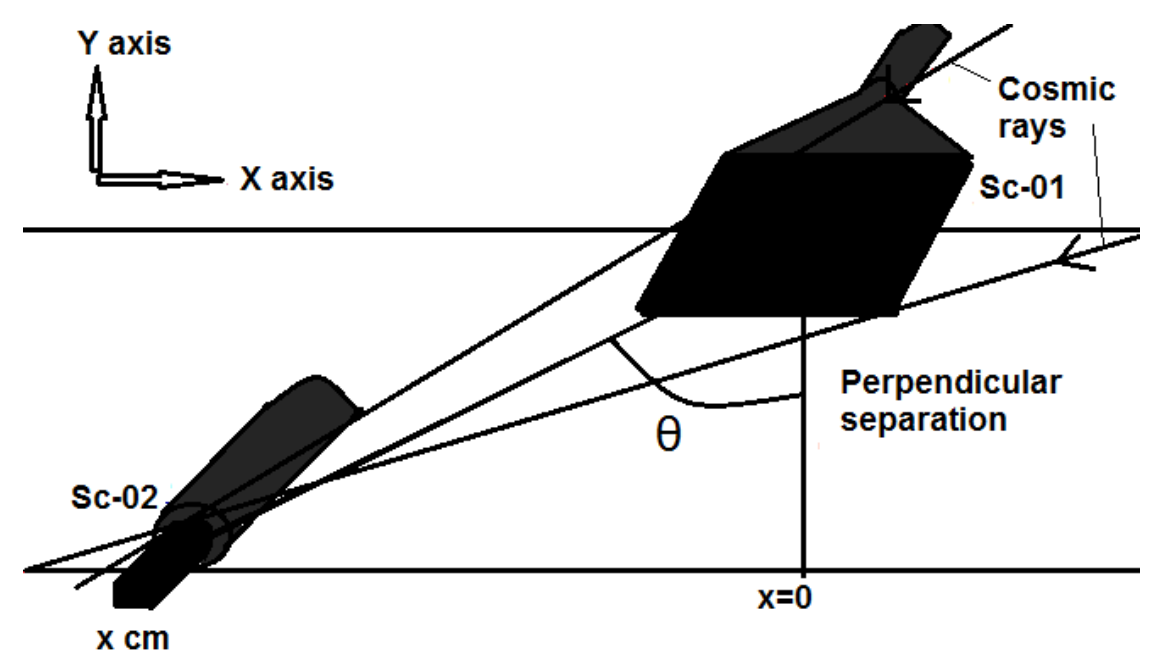

Figure 9: Schematic arrangement of Sc-01 and Sc-02 to study the angular variation of cosmic rays

and the coincidence counts are normalised by $\Omega(\theta)$. Counts are measured for a time duration of 60 minutes for each setting. The coincidence count rate per unit solid angle per unit area as a function of the zenith angle is shown in Figure [س. It is seen from Figure 四 that the cosmic ray intensity decreases from $\sim 7 \times 10^{-3}$ to $3 \times 10^{-3} \mathrm{~s}^{-1} \mathrm{~cm}^{-2} \mathrm{sr}^{-1}$ corresponding to a zenith angle of $0^{\circ}$ to $70^{\circ}$ respectively.

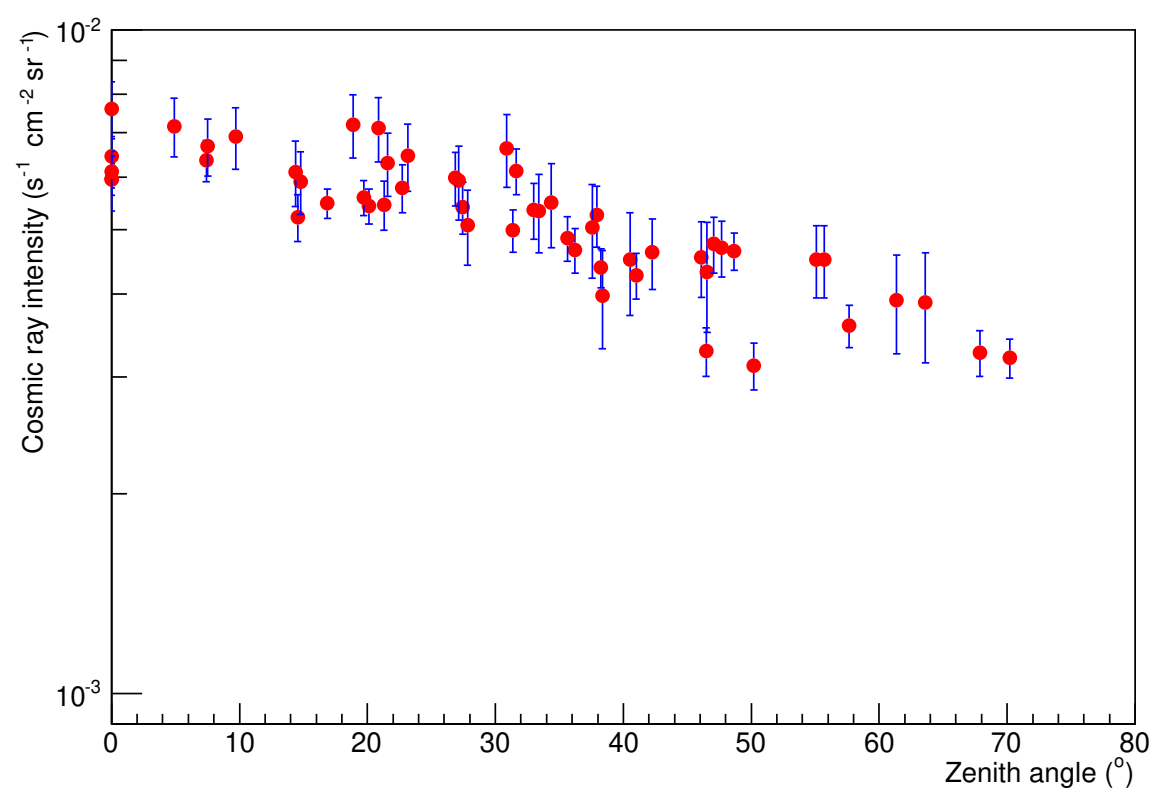

Figure 10: Variation of the cosmic ray intensity with the zenith angle 


\section{Cosmic ray air shower array at Darjeeling}

A hexagonal array consisting of 7 plastic scintillator detectors is built to study cosmic ray air showers at an altitude of about $2200 \mathrm{~m}$ a.s.l. in the Eastern Himalayas (Darjeeling, $27^{\circ} 3^{\prime} \mathrm{N} 88^{\circ} 16^{\prime} \mathrm{E}$ ) as described in Ref. [15]. Six detectors are placed at the vertices of the hexagon and one at the centre. The separation between two detectors is $8 \mathrm{~m}$ and the array covers an area of $168 \mathrm{~m}^{2}$. Each detector element consists of four plastic scintillators of dimension $50 \mathrm{~cm} \times 50 \mathrm{~cm} \times 1 \mathrm{~cm}$ making the total active area of $1 \mathrm{~m} \times 1 \mathrm{~m}$. These plastic scintillators are fabricated indigenously at the Cosmic Ray Laboratory (CRL), TIFR, Ooty, India [6, Z, [21]. All four scintillators of a detector are coupled with a single PMT using a bundle of 48 wavelength shifting (WLS) fibers spread over the active area of the module. Each detector is kept in an aluminium box and only two connectors, one for the application of high voltage (HV) and one for the signal collection, are fixed to each of them. Each box is sealed using silicone rubber and kept on a metal stand in the open air. The schematic of the

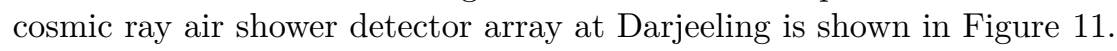

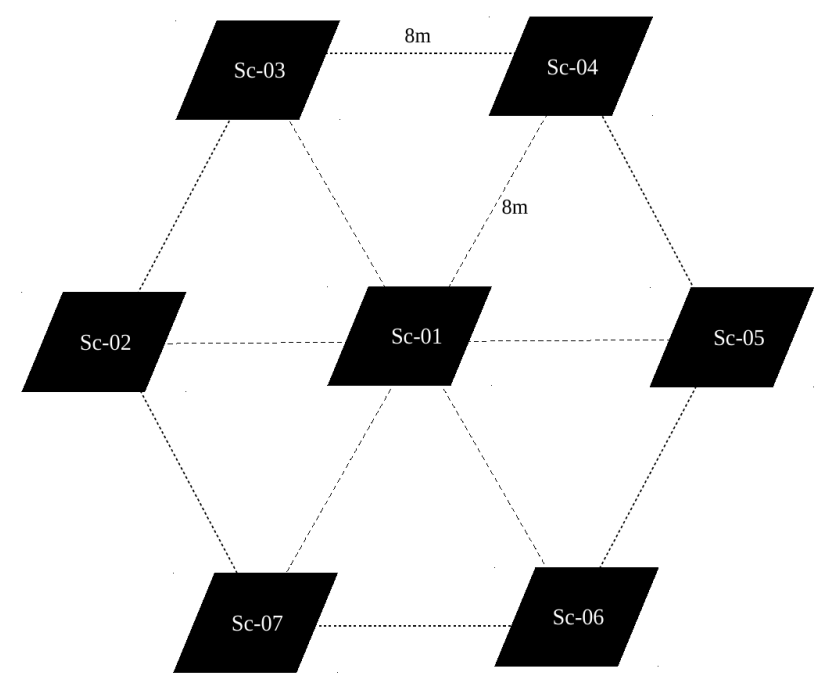

Figure 11: Schematic of the hexagonal cosmic ray air shower detector array at Darjeeling

\subsection{Fabrication of detectors}

We have built an array of active detectors to detect cosmic ray air showers at an altitude of about 2200 meters a.s.l. in the Himalayas. Each of the elements of this array is a $1 \mathrm{~m} \times 1 \mathrm{~m}$ plastic scintillator coupled with WLS fibers and PMT [1.9, 201].

All $1 \mathrm{~m} \times 1 \mathrm{~m}$ scintillators are made of four $50 \mathrm{~cm} \times 50 \mathrm{~cm}$ blocks of thickness $1 \mathrm{~cm}$. The scintillators are of the same type as used in Ref [Z]]. All four scintillator blocks are connected to a single PMT by using circular WLS optical fibers (Kuraray scintillating fiber). 12 parallel grooves are made in a $0.5 \mathrm{~m} \times 0.5 \mathrm{~m}$ block to place the WLS fibers as shown in figure 4-a of Ref [20]]. Total 48 WLS fibers from all 4 blocks are collectively coupled optically to a PMT model ETL9807B of diameter $5 \mathrm{~cm}$. A few steps of fabrication of the detector are shown in Figure [2. Four scintillator blocks covered by black sheets along with the WLS optical fiber and PMT is shown in Figure [2. There are no optical contacts between the four scintillator blocks. The whole system is kept in an aluminium box for light tightness. The PMT is mechanically fixed inside the box and connected to the base circuit. The negative HV to the PMTs is applied using MHV cable and the signal is collected by BNC cable. The schematic diagram of a detector module is shown in Figure [13. Initially, all the PMTs are calibrated and their individual efficiencies are measured using other scintillator blocks of the same kind.

One typical muon signal from one scintillator detector taken from the oscilloscope is shown in Figure प4. The typical signal amplitude is $\sim-200 \mathrm{mV}$ and rise time $\sim 5 \mathrm{~ns}$ at $-1500 \mathrm{~V}$ as shown in Figure प4.

Keeping the applied voltage constant at $-1725 \mathrm{~V}$ for all three scintillator detectors singles count rates are measured varying the threshold voltage and it is found that $-40 \mathrm{mV}$ is enough to cut all the noise. With $-40 \mathrm{mV}$ threshold settings the three-fold coincidence of any three such detectors give a cosmic ray muon flux of about 1.2 per min per $\mathrm{cm}^{2}$ at an altitude of about 2200 meters a.s.l. 

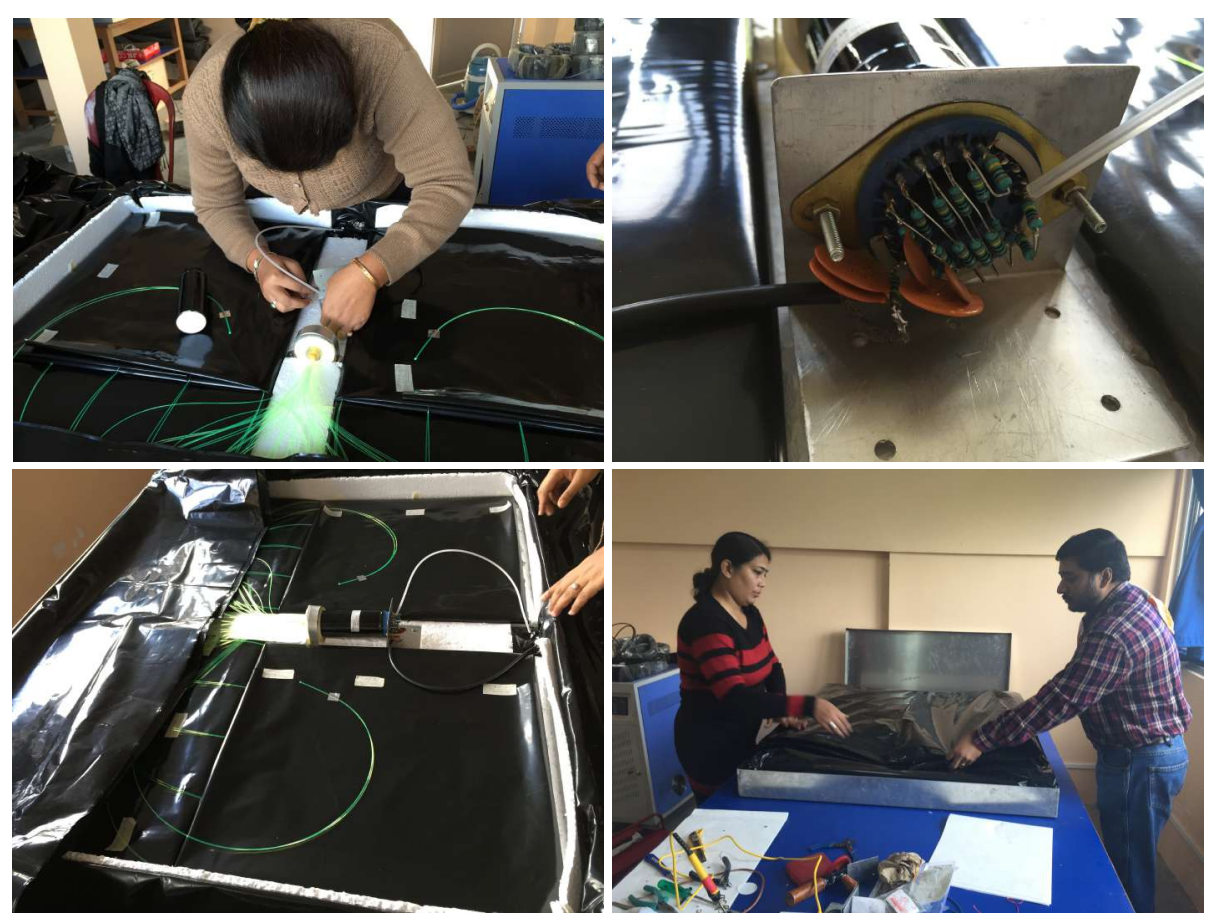

Figure 12: (Top left) WLS fiber insertion, (Top right) Voltage divider network, (Bottom left) HV cable connected in proper position, (Bottom right) Closing the box

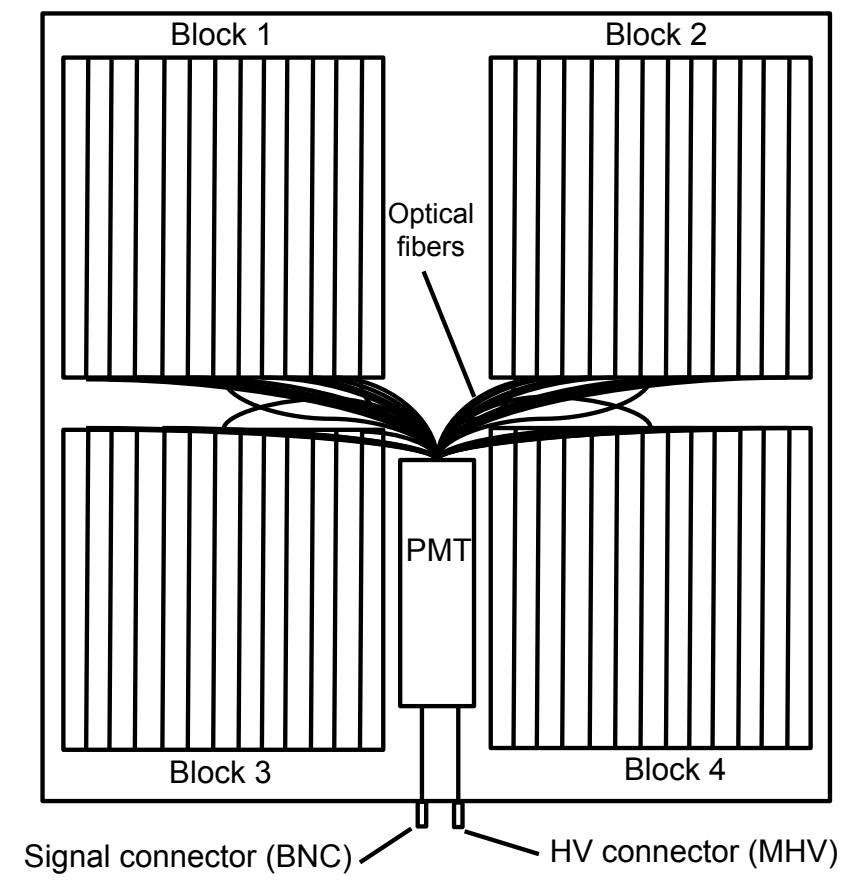

Figure 13: Block diagram of a detector module

\subsection{Preliminary test in the lab}

Initially, in the lab, four detectors named as scintillator 1, scintillator 2, scintillator 3 and scintillator 4 are built. Of them three detectors are used to mimic the cosmic ray shower. After fabricating the detectors each detector is tested varying the applied negative $\mathrm{HV}$ and measuring the singles count rate per unit area. 


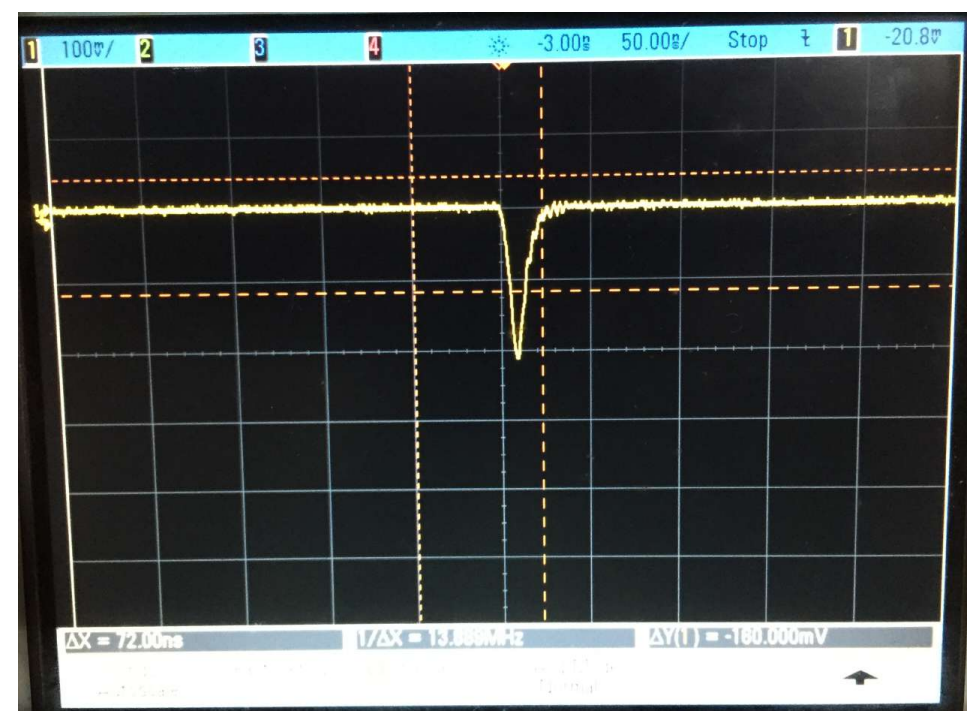

Figure 14: Typical muon signal at $-1500 \mathrm{~V}(100 \mathrm{mV} / \mathrm{Div}, 50 \mathrm{~ns} / \mathrm{Div}, 50 \Omega$ load $)$

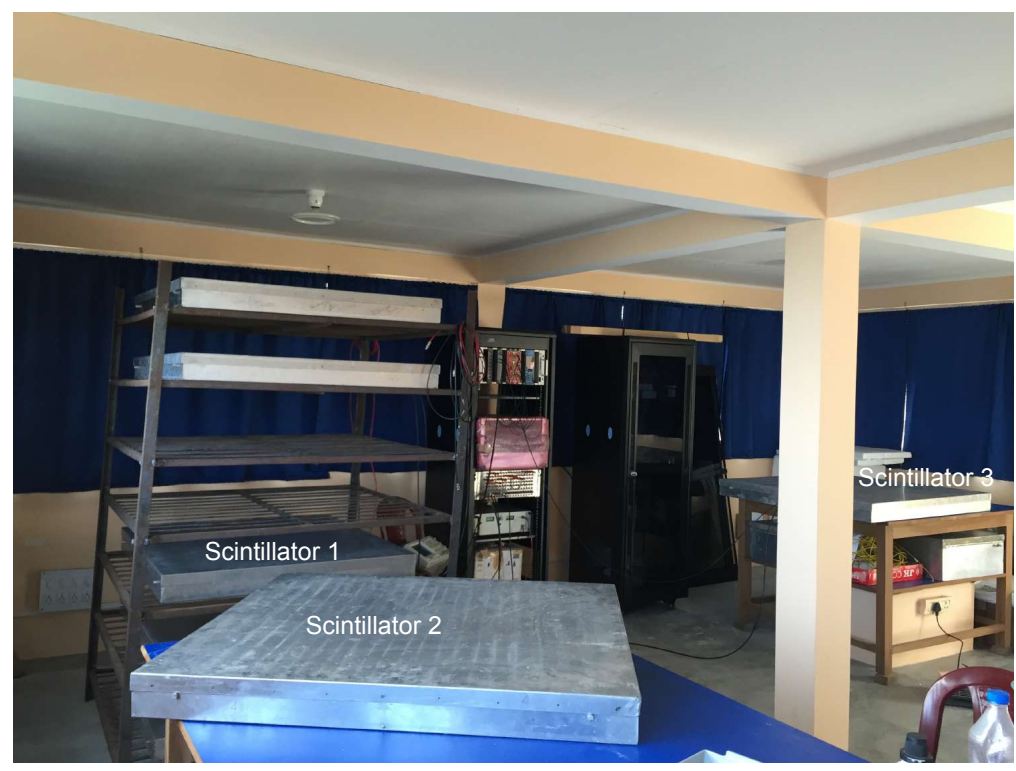

Figure 15: Horizontal arrangement of three scintillator detectors to measure cosmic ray shower

Firstly the scintillators are placed in a vertical stack to detect the coincidence signal of cosmic ray muons. From the stack, the muon detection efficiency of a particular detector is measured using a coincidence trigger from the other three modules. The trigger is taken from the three fold coincidence of three scintillators. The trigger width is kept fixed at $400 \mathrm{~ns}$. The efficiency is defined as the ratio of four-fold coincidence and three fold coincidence trigger.

As a preliminary test, to mimic the cosmic ray shower, three scintillator detectors are placed on a horizontal plane. A horizontal arrangement of three scintillator detectors to measure cosmic ray shower is shown in Figure [5. The centres of the scintillator detectors make a triangle of sides $1.9 \mathrm{~m}, 3 \mathrm{~m}$ and $3.5 \mathrm{~m}$ respectively. $-1725 \mathrm{~V}$ is applied to all three detectors and threshold to the leading edge discriminator (LED) module is set at $-40 \mathrm{mV}$ for all three. The width of the discriminated NIM signal is fixed at $150 \mathrm{~ns}$ for each module. The three-fold coincidence from this horizontal arrangement of three detectors, which mimics a cosmic ray shower, is measured for about 1 month period. Each reading is taken for one hour duration.

For three scintillators the singles counting rate per unit area as a function of applied HV is shown in Figure ए6]. 


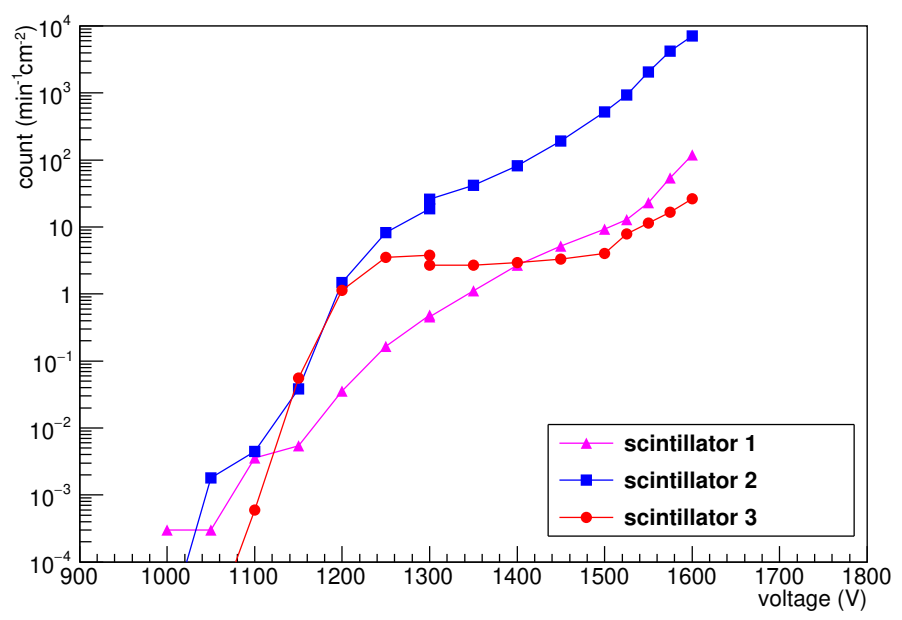

Figure 16: Count rate per unit area as a function of applied voltage

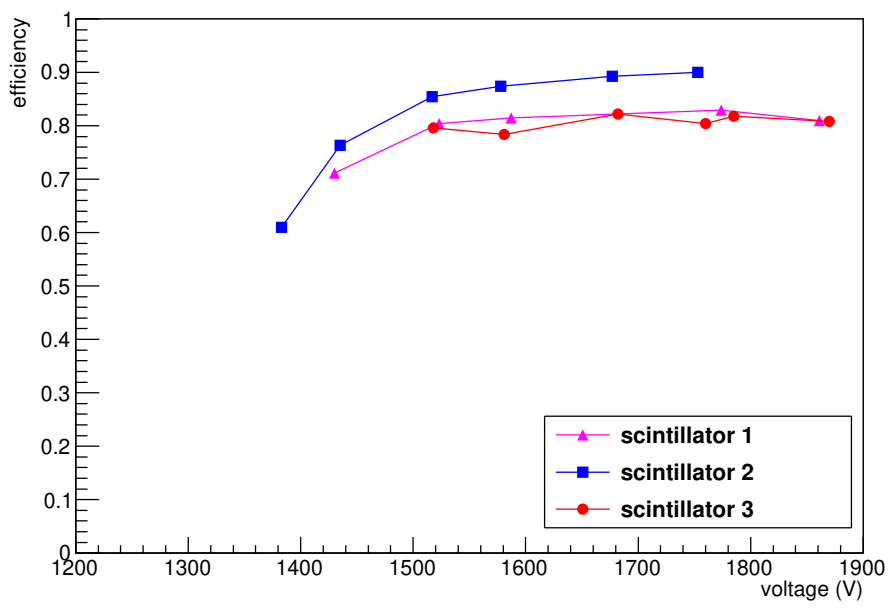

Figure 17: Efficiency as a function of applied voltage

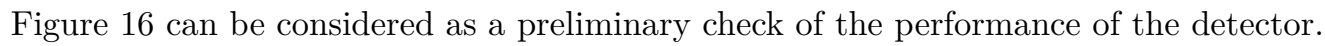

As described in section $\mathbf{B 2}$ the efficiency of the three scintillator detectors as a function of applied HV is measured and shown in Figure 므. It is seen from Figure $\$$ that for all three detectors the efficiency reaches a plateau at a value greater than $80 \%$ and the voltage regions are nearly the same for all modules. The applied voltage is increased to $-1850 \mathrm{~V}$ to see the efficiency plateau. The value of efficiency is a little different for different modules. This is because of different area coverage of trigger detectors as explained next. The detectors are placed vertically for these measurements. A trigger is taken by the coincidence signal of the three detectors. In such a case the efficiency measured for the detector, which is placed in the middle of the trigger set-up will give the best result, because the particle that is detected by the trigger detector must pass through the fourth (in the middle) module. For the other two cases trigger from three detectors (either placed on top or bottom of the detector under study) may leave the system without passing through the fourth module resulting a decrease in efficiency.

As a proof of principle, to mimic a cosmic ray shower array the three-fold coincidence of three scintillators placed horizontally is demanded as stated in section $\mathbf{3 2}$. $-1725 \mathrm{~V}$ is applied to all three detectors and the cosmic ray shower is counted for a period of one month during the middle of November to the middle of December 2016. The result is shown in Figure 미. It is observed that the shower rate varies with time between $0.25-0.35$ 


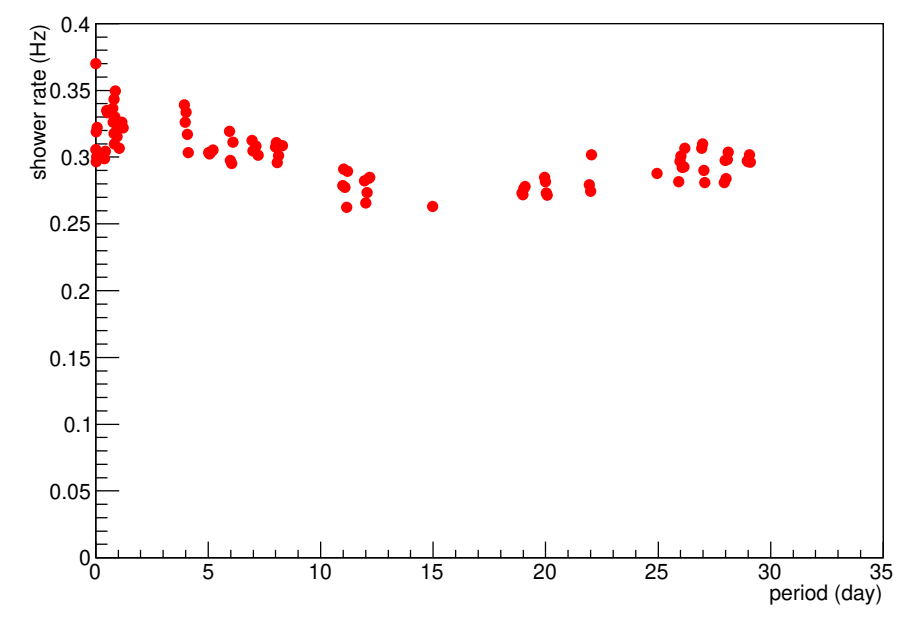

Figure 18: Shower rate vs. time during mid November - mid December, 2016

$\mathrm{Hz}$ in this period. This mimics a cosmic ray air shower.

\subsection{Observation and Results}

After successful operation of three detectors and building the remaining modules each box is sealed using silicone rubber and kept on a metal stand in the open air to complete the array. The real array at Darjeeling is shown in Figure पप.

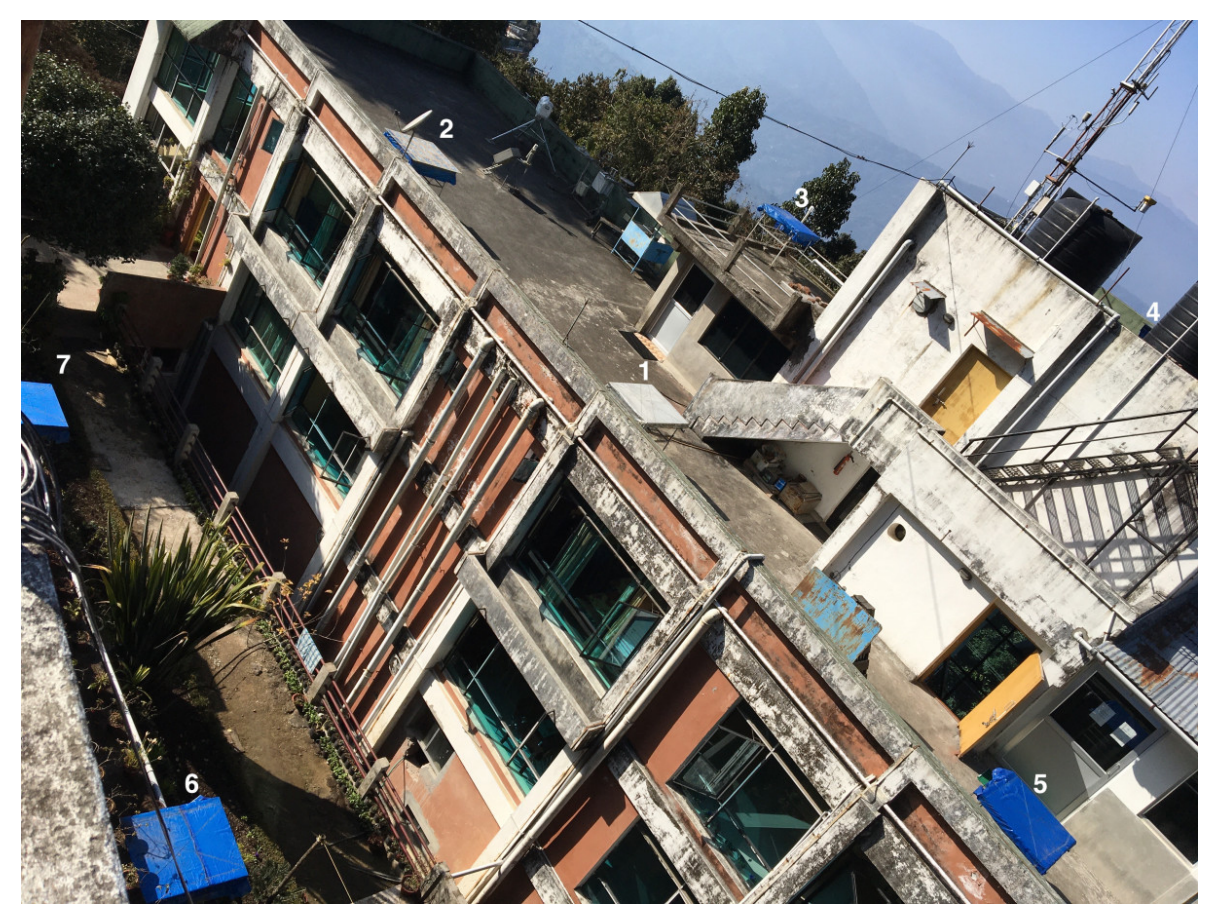

Figure 19: Cosmic ray air shower detector array at Darjeeling

From the electronics rack kept inside the counting room, the length of cables for the signal collection are made $50 \mathrm{~m}$ for all the modules to eliminate the time delay in signals due to the different cable lengths. The length of HV cables are also made $50 \mathrm{~m}$ for all the modules. All detectors are biased with - $1740 \mathrm{~V}$ from a single 
HV power supply using an external HV distribution network. The signals from the detectors are fed to the LED and a common threshold of $-20 \mathrm{mV}$ is set to all. It is observed that - $20 \mathrm{mV}$ threshold is enough to eliminate the noise. A custom-built module, developed at TIFR, with seven inputs is used to generate the multi-fold trigger. Seven individual signals from the LED are fed to the trigger module. The shower trigger is generated when the central detector and any two other scintillator detectors give signal simultaneously. The NIM output signal from the trigger module is counted using a scaler module also fabricated at TIFR. For some period the 7-fold coincidence signal from all the scintillator detectors is also counted to measure large shower. The trigger output and the 7-fold logic signals are counted for 60 minutes to get each data point. The counts are made so far manually. It has been observed that in 60 minutes a significant number of counts are accumulated.

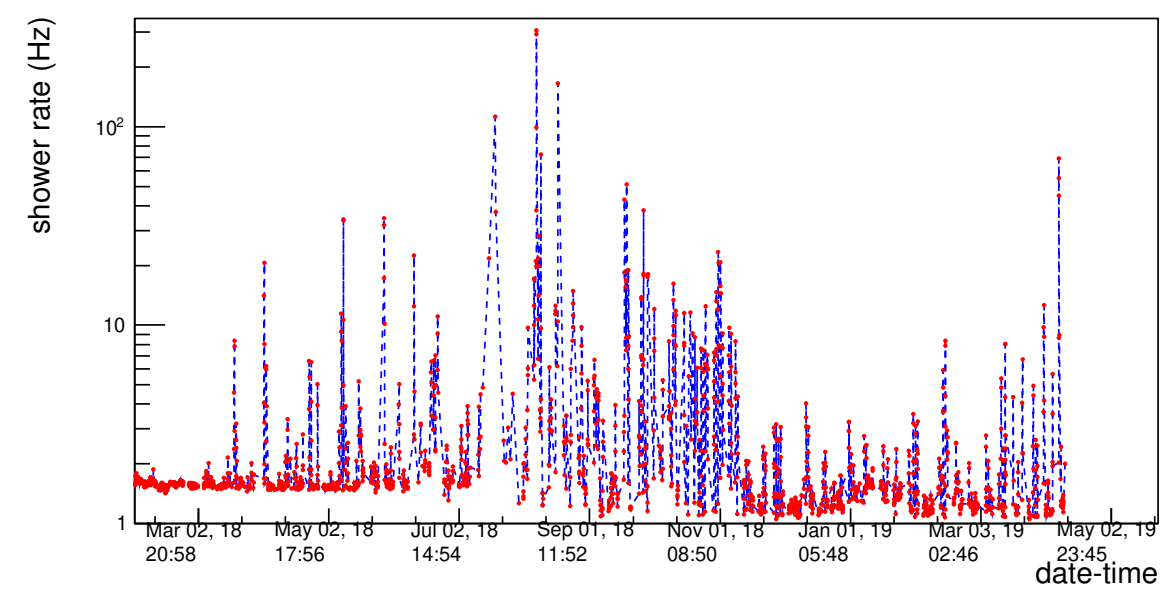

Figure 20: Cosmic ray air shower rate as a function of date and time. Data from February 2018 to May 2019 is presented. Each data point is the average count rate measured in 60 minute duration.

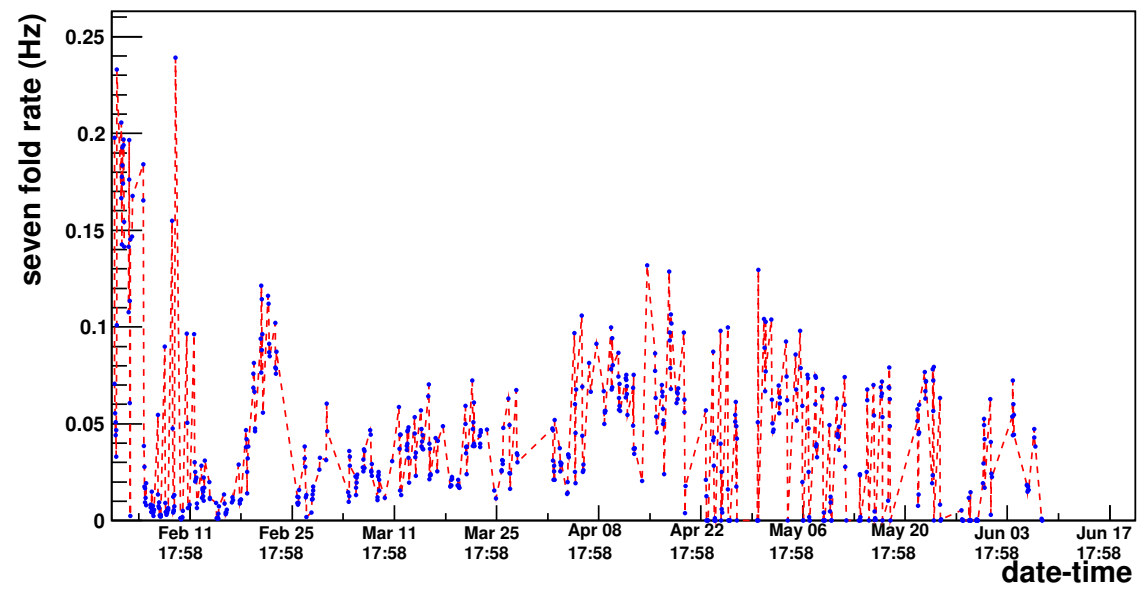

Figure 21: 7-fold coincidence rate as a function of date and time. Data from February 2018 to June 2018 is shown. Each data point is the average count rate measured in 60 minute duration.

Continuous measurement of cosmic ray air shower i.e. the output of the trigger module and also the large shower i.e. the 7 -fold coincidence count (for some period) is done since the end of January 2018. Each data is taken for a duration of 60 minutes. The shower rate and the 7 -fold coincidence rate as a function of date-time is shown in Figures $[\mathbb{Z}$ and $2 \mathbb{U}$ respectively. From the measurement (Figure $20 \mathrm{U}$ ) it is observed that the shower rate shows some significantly high values on some days. It has been noted from other literature that there were either some solar flares or proton fluence, electron fluence, increase of $K_{p}$ index on these days [21, [22, 2:3]. It is 


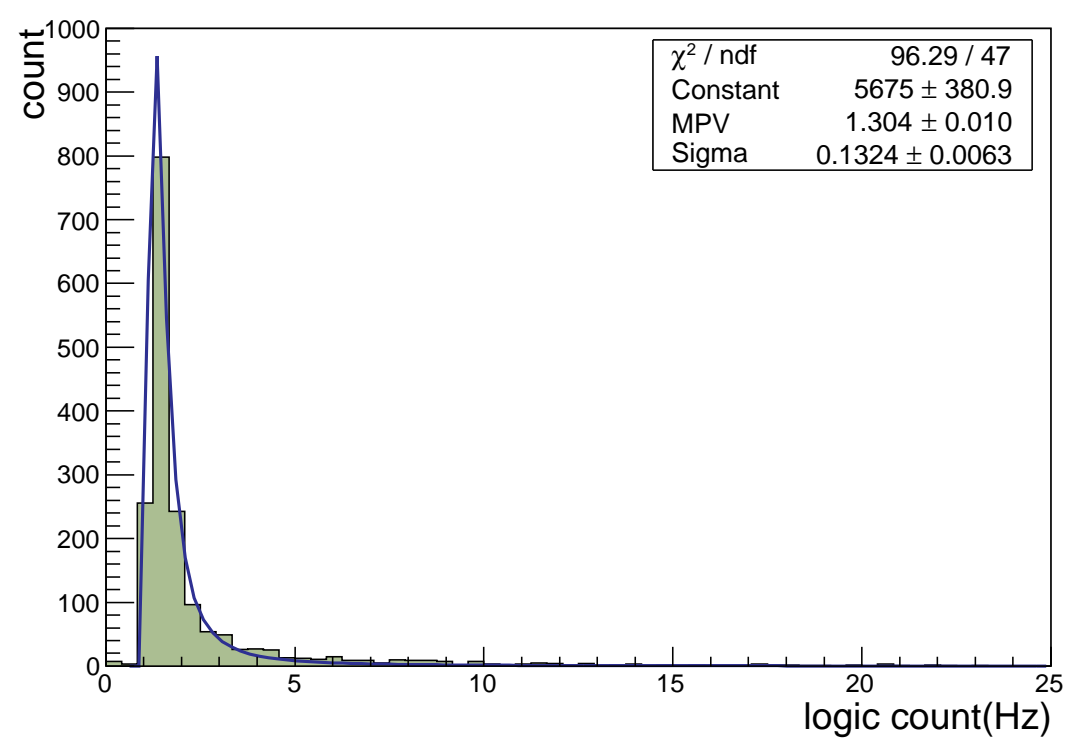

Figure 22: Distribution of shower rate

also to be mentioned here that all the spikes obtained are around afternoon (12:00 - 15:00 hours) of those days. However, such behaviour is not present in the measurement of 7 -fold coincidence rate (Figure $2 \mathbb{}$ ).

The distribution of the shower rate is fitted with a Landau function as shown in Fig. ए2. From a distribution of shower rate, it is found that the most probable value of the air shower rate, excluding the high values, is $\sim 1.3 \mathrm{~Hz}$ with a sigma of 0.13 . The 7 -fold coincidence rate as measured for some period is found to be $\sim 0.04 \mathrm{~Hz}$ with an RMS of 0.02 . This value could be compared with that obtained from a large array at a similar altitude but different geographical locations [24].

\section{Summary and outlooks}

In this review, I have summarised the R\&D activities carried out in the detector laboratory of Bose Institute, Kolkata, and Darjeeling Campus of Bose Institute on the scintillation detectors for the cosmic ray experiments. This program includes mostly cosmic ray studies with plastic scintillators. This is an observational research program.

In the Kolkata campus, after fabricating the detectors the count rates are measured with cosmic rays, $\mathrm{Co}^{60}$, $\mathrm{Cs}^{137}$ and $\mathrm{Na}^{22}$ sources. The angular variation of cosmic ray intensity is measured, in Kolkata, India during the beginning of 2017, with the coincidence technique and it is observed that the cosmic ray intensity decreases from $\sim 7 \times 10^{-3}$ to $3 \times 10^{-3} \mathrm{~s}^{-1} \mathrm{~cm}^{-2} \mathrm{sr}^{-1}$ corresponding to an increasing zenith angle of $0^{\circ}$ to $70^{\circ}$ respectively. This result is a little bit different both in absolute magnitude and the angular variation as reported in Ref. [एD]. Actually, the cosmic ray intensity at sea level is a quantity that varies with the geomagnetic latitude, altitude, solar activity and atmospheric conditions. When comparing cosmic ray flux at low energies $(<20 \mathrm{GeV} / \mathrm{c})$ it is important to know the year and location where the measurements are made.

The same type of measurements with scintillators of larger dimension and taking coincidence with more than two scintillators are in future plan. The study of east-west asymmetry of cosmic muons will also be carried out.

An array of seven plastic scintillator detectors is commissioned and operational since the end of January, 2018, for detection of cosmic ray air showers at an altitude of about 2200 meters a.s.l. in the Himalayas at the Centre for Astroparticle Physics \& Space Sciences, Darjeeling campus of Bose Institute. From this array, it is found that at an altitude of about $2200 \mathrm{~m}$ the most probable value of shower rate is $\sim 1.3 \mathrm{~Hz}$ with a sigma of 0.13. 7 -fold coincidence rate is found to be $\sim 0.04 \mathrm{~Hz}$ with an RMS of 0.02 in the initial period. In the shower rate plot (Figure $\mathbf{2 0}$ ) it can be observed that on some days the shower rate is significantly high. It is found that there was either some solar flares or proton fluence, electron fluence, increase of $\mathrm{K}_{p}$ index on these particular days [211, [22, [23]. 
So far the detector systems are operated using a NIM electronics system where the data acquisition is done manually during day time only (Morning 7 a.m. to Evening 6 p.m. Indian Standard Time (IST)). Most of the jumps are observed during 12:00-15:00 hours.

Since the installation is located at a relatively high altitude $(2200 \mathrm{~m})$ so the detector can also be made more sensitive to the primary radiation with respect to the similar installation at sea level. We are also planning on estimating the energy of the primary cosmic ray that initiated the shower we are measuring with the detector. We are also working on the integration of the array with a CAMAC based DAQ which will enable recording the data automatically.

This small array is a pilot project. After completion of phase I of this project, the objective will be to set up the complete mini-array of 64 such scintillator detectors coupled with WLS fiber and PMT, at the Darjeeling campus of Bose Institute.

\section{Acknowledgements}

Firstly, I would like to thank my students Ms. Shreya Roy, Mr. Sayak Chatterjee and Mr. Arindam Sen for doing the work meticulously, taking data, analysing data and making plots.

I would like to thank Mr. Sayan Chakraborty, Mr. Shibnath Shaw, Ms. Nilanjana Nandi, Mr. Shubham Jaiswal, Ms. Aayushi Paul and Mr. Shivsant Chauhan for some valuable measurements.

I would like to thank the scientists of Cosmic Ray Laboratory (CRL), Tata Institute of Fundamental Research (TIFR), Ooty, India for fabrication of the scintillators indigenously for the cosmic ray shower array. I would also like to thank Bose Institute workshop for all the mechanical work. We are thankful to Mrs. Sumana Singh and Mr. Subrata Das for assistance in the assembly of the detector modules. We are also thankful to Mr. Deb Kumar Rai, Mrs. Yashodhara Yadav, Mr. Sabyasachi Majee, Mr. Vivek Gurung for their help in the course of this work. Thanks to Mrs. Sharmili Rudra of Seacom Engineering College for some important measurements after building the scintillator detectors at Darjeeling. Sincere thanks to the efforts made by Ms. Debonita Saha of St. Xaviers College for formatting the raw data from detectors. I would like to thank Dr. C.J. Schmidt and Mr. Jörg Hehner of GSI Detector Laboratory and Mr. Dipanjan Nag, Mr. Rathijit Biswas, Dr. Atanu Maulik, Prof. Sanjay K. Ghosh, Prof. Sibaji Raha, Prof. Supriya Das, Prof. Rajarshi Ray, Dr. Sidharth K. Prasad, Dr. R. P. Adak, Dr. Abhijit Chatterjee and Prof. Somshubhro Bandyopadhyay of Bose Institute for valuable discussions and suggestions in the course of all the studies. I also acknowledge the support of DST-SERB Ramanujan Fellowship (D.O. No. SR/S2/RJN-02/2012). Finally, I acknowledge the IRHPA Phase-II project (IR/S2/PF-01/2011 Dated 26/6/2012) of Department of Science and Technology, Government of India.

\section{References}

[1] A. Sen et al., Pramana - J. Phys. (2021) 95:64.

[2] M. Aglietta et al., The EAS-TOP array at Gran Sasso: results of the electromagnetic detector, Nucl. Phys $B \mathbf{1 6}$ (1990), 493.

[3] M. Aglietta et al., Detection of the UHE Burst from the Crab Nebula on February 23, 1989, from the EAS-TOP Array, Europhysics Letters 115 (1991) 81.

[4] KASCADE Collaboration, Electron, muon, and hadron lateral distributions measured in air showers by the KASCADE experiment, Astroparticle Physics 14 (2001) 245.

[5] W.D. Apel et al., The KASCADE-Grande experiment, Nucle. Instr. Meth. A 620 (2010) 202.

[6] S.K. Gupta et al., The current status of the GRAPES-3 extensive air shower experiment, Nuclear Physics $B$ - Proceedings Supplements 196 (2009) 153M.

[7] S.K. Gupta et al., GRAPES-3 A high-density air shower array for studies on the structure in the cosmic-ray energy spectrum near the knee, Nuclear Instruments and Methods in Physics Research A 540 (2005) 311.

[8] Mehmet Bektasoglu and Halil Arslan, Pramana - Journal of Physics, Vol. 80, No. 5, May 2013, pp 837 - 846.

[9] S. Roy et al., Proceedings of ADNHEAP 2017, Springer Proceedings in Physics 201, ISBN 978-981-10-7664-0. 
[10] A.N. Dmitrieva et al., 29th International Cosmic Ray Conference Pune (2005) 6, 73-76, [arXiv:hepex/0611051v1].

[11] Alexander Mishev, arXiv: physics/0602120 v.2 .

[12] S. Cecchini and M. Spurio, Geosci. Instrum. Method. Data Syst., 1, 185-196, 2012, [arXiv:1208.1171v1].

[13] ROOT, A Data Analysis Framework, CERN, website: <http://www.root.cern.ch>.

[14] S. Biswas et al., 2017 JINST 12 C06026.

[15] S. Roy et al., Nucl. Instrum. Methods A 936 (2019) 249.

[16] S. Roy et al., Development of an Extended Air Shower array at Darjeeling : an update, Proceedings of XXIII DAE High Energy Physics Symposium, (To be published).

[17] G. F. Knoll, Radiation Detection and Measurement, (3 ${ }^{\text {rd }}$ Edition).

[18] W. R. Leo, Techniques for Nuclear and Particle Physics Experiments, (2 ${ }^{\text {nd }}$ Edition).

[19] P.K. Mohanty, S.R. Dugad, S.K. Gupta, Monte Carlo code G3sim for simulation of plastic scintillator detectors with wavelength shifter fiber readout, Review of Scientific Instruments 83 (2012) 043301.

[20] P.K. Mohanty et al., Measurement of some EAS properties using new scintillator detectors developed for the GRAPES-3 experiment, Astroparticle Physics 31 (2009) 24.

[21] https://www.spaceweatherlive.com/en/solar-activity/top-50-solar-flares/year/2018

[22] ftp://ftp.swpc.noaa.gov/pub/forecasts/SGAS/

[23] http://tesis.lebedev.ru/en/forecast_activity.html?m=3\&d=19\&y=2018

[24] M. Zuberi et al., PoS (ICRC2017) 302. 